\title{
Pollen-inspired Synthesis of Porous and Hollow NiO Elliptical Microstructures Assembled from Nanosheets for High-Performance Electrochemical Energy Storage
}

Shaolan Wang ${ }^{\mathrm{a}}$, Wei $\mathrm{Li}^{\mathrm{a}}$, Lipeng Xin ${ }^{\mathrm{a}}$, Ming Wu ${ }^{\mathrm{a}}$, Wenping Sun ${ }^{\mathrm{b}}$, Xiaojie Lou ${ }^{\mathrm{a}^{*}}$

${ }^{a}$ Frontier Institute of Science and Technology, and State Key Laboratory for

Mechanical Behavior of Materials, Xi'an Jiaotong University, Xi'an, 710049,

\section{P.R.China}

${ }^{b}$ Institute for Superconducting and Electronic Materials, University of Wollongong, Wollongong, NSW 2522, Australia

*Corresponding author.

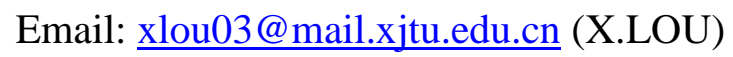


Abstract

Numerous efforts have recently been focused on designing and synthesizing efficient nanostructures electrode materials for high-efficiency energy storage. Herein, hierarchical porous and hollow $\mathrm{NiO}$ elliptical microstructures constructed from nanosheets are synthesized by utilizing the renewable, cost-effective and widespread native rape pollen grains as template. Benefiting from the unique morphology, pollen-templated $\mathrm{NiO}$ shows outstanding electrochemical performances as electrode of supercapacitor, such as high specific capacitance (198.7 $\mathrm{mAh} \mathrm{g}^{-1}$ at $\left.1 \mathrm{~A} \cdot \mathrm{g}^{-1}\right)$, excellent rate capability (89.2 $\mathrm{mAh} \mathrm{g}^{-1}$ at $\left.30 \mathrm{~A} \cdot \mathrm{g}^{-1}\right)$ and long cycling life. Besides, asymmetric supercapacitor device was assembled by using pollen-templated $\mathrm{NiO}$ and activated carbon, which deliver excellent cycling stability (103.3\% retention after 10000 cycles) as well as high energy/power density (a high energy density of $66.4 \mathrm{Wh} \mathrm{kg}^{-1}$ and power density of $22.2 \mathrm{~kW} \mathrm{~kg}^{-1}$ ). Moreover, the low-cost pollen template-based strategy is found to be a versatile method to prepare other porous and hollow elliptical microstructure of transition-metal oxides.

Keywords: pollen-templated $\mathrm{NiO}$, hollow and porous structure, asymmetric supercapacitor 


\section{Introduction}

The energy capture and related environment issues have becoming the pivotal constraint for the development of human society.[1,2] Consequently, there has been a growing concern in developing renewable energy storage and conversion devices in the past decades.[3] In particular, supercapacitors, with appealing high power density and reliable cycling stability, have been considered as one of the most promising energy storage systems. $[4,5]$ Since the performances of supercapacitors mainly depend on its electrode material, substantial effort has been made to design novel electrode materials with desirable capacitive properties.[6, 7] Significantly, transition metal oxides have been widely utilized in supercapacitors due to its high theoretical specific capacitance, diverse crystallographic construction and variable valance state, etc. [8, 9] Among them, $\mathrm{RuO}_{2}$ has been widely researched on account of their high proton conductivity and excellent reversible redox process.[10, 11] However, the low reserve of earth resources, toxicity and high cost have limited its large-scale application.[12, 13]

In this case, earth-abundant Ni-based alloys are good substitutes for replacing Ru-based supercapacitors. Particularly, the unique p-type semiconductor $\mathrm{NiO}$ is a promising electrode material due to its high theoretical capacity, high redox activity and chemical stability, environment-friendly nature and low cost.[14-16] Despite the extensive exploration of NiO-based supercapacitor, the specific capacitance is far from the theoretical values.[17, 18] Although some of the reported $\mathrm{NiO}$ could reach a high specific capacitance, unfortunately, the achievement in specific capacitance often be at the expense of other electrochemical performances, such as the worse rate 
capability, instability in the long time cycling test and limited power/energy density. Therefore, attempts to pursue high comprehensive electrochemical in single electrode remains even more challenging.

Interestingly, recent studies demonstrate that the electrochemical performance of NiO-based supercapacitors could be improved by tailoring multifunctional nano-architecture engineering (nanowires,[19] nanosheets,[20] nanodots,[21] nanoflake,[22] hierarchical nanostructures,[23] hollow structures,[24] etc.). Among them, porous/ hollow morphology is an effective approach to enhance the electrochemical properties of NiO.[25] This distinctive microstructure can lead to the efficient improvement in electron/ion conductivity, diffusion and mass transport between the electrolyte and electrode materials, facilitate the electron transfer and provide more electrochemical active sites for the enhanced redox reaction, therefore improving the electrochemical performance.[26]

Generally, one of the straightforward methods to synthesize porous and hollow structures is template-based synthesis.[27-29] In addition to the common templates (such as surfactant, polymer, virus, etc.), nature has provide some valuable templates.[30] By precisely control and replication, natural biotemplates have shown superiority in obtaining materials with controllable multifunctional bio-structures.[31] Among a variety of outstanding biotemplates (such as diatom,[32] butterfly wing,[33] grapefruit exocarp,[34] rice husk,[35] cotton fiber,[36] banana peel,[37] legume,[38] etc.), rape pollen grains have drawn much attention for their unique three-dimensional porous and hollow structure.[39, 40] However, there is very limited research on 
synthesizing electrode materials with porous/hollow structure by using pollen grains as template.

In this work, porous and hollow $\mathrm{NiO}$ elliptical microstructures assembled from nanosheets was prepared by using natural rape pollen grains as template. Benefiting from the unique morphology, the pollen-templated $\mathrm{NiO}$ exhibited high specific capacitance, excellent rate capability and long cycling life in both three electrode system and asymmetric device. Meanwhile, pollen-templated hollow $\mathrm{SnO}_{2}$ and $\mathrm{TiO}_{2}$ elliptical microstructures were also synthesized, demonstrating the universality of this method to synthetize porous/hollow microstructures.

\section{Experimental}

2.1 Pretreatment of pollen grains: All the reagents used in this work were of analytical grade without further purification. Pollen grains were pretreated by a novel method. In a typical synthesis, $6.0 \mathrm{~g}$ natural rape pollen grains (Pollen-A) were immersed in $150 \mathrm{~mL}$ anhydrous ethanol with ultrasonication for $60 \mathrm{~min}$ before filtered and washed with a large amount of deionized water (DI). Then, a mixed solution containing glacial acetic acid $(12.5 \mathrm{~mL})$ and alcohol $(37.5 \mathrm{~mL})$ was added into and kept at room temperature for $90 \mathrm{~min}$. After pretreatment, part of core materials (proteins, amino acids, vitamins and nucleic acids, etc.) were removed, showing the hollow structure. Then, the samples were collected by filtration, rinsed with deionized water until $\mathrm{pH}=7$ before drying at $60{ }^{\circ} \mathrm{C}$ overnight, named as Pollen-B.

Preparation of the rape pollen-templated 3D NiO nanosheets: The synthesis process of pollen-templated $\mathrm{NiO}$ (named as $\mathrm{P}-\mathrm{NiO}$ ) including the chemical bath 
deposition and the subsequent calcination. Typically, $0.2 \mathrm{~g}$ Pollen-B were added into a mixed solution $(40 \mathrm{~mL}$ of $0.2 \mathrm{M}$ nickel acetate and $30 \mathrm{~mL}$ of $0.05 \mathrm{M}$ potassium persulfate solution) and magnetically stirred for $40 \mathrm{~min}$. Subsequently, $4 \mathrm{~mL}$ aqueous ammonia was added into the above solution drop by drop. The chemical bath deposition process was carried out for 30 min under constant stirring. Afterwards, the obtained powders were filtered, rinsed with deionized water and ethanol before dried at $60{ }^{\circ} \mathrm{C}$ overnight in vacuum oven. Finally, P-NiO powders were obtained after annealing in a tube furnace in air at $500{ }^{\circ} \mathrm{C}$ for $2 \mathrm{~h}$ with a heating ramp of $5{ }^{\circ} \mathrm{C}$ per min. Moreover, for comparison, $\mathrm{NiO}$ without the addition of rape pollen grains (W-NiO), and Pollen-B after calcination (named hereafter as Pollen-C) were also prepared under the same experimental condition.

2.2 Materials characterization: The crystalline structure of samples was characterized by X-ray diffraction (XRD, SHIMADZU, XRD-7000). Field-Emission Scanning Electron Microscopy (FESEM, Hitachi, SU-8010) and Energy Dispersive Spectroscopy (EDS) was used to characterize the morphology and composition of the products. Transmission Electron Microscopy (TEM) measurements were taken on a JEM 2100F microscopy. Thermogravimetric analysis (TGA) was conducted on TGA/DSC1 (Mettler Toledo) with a heating rate of $10{ }^{\circ} \mathrm{C} \min ^{-1}$ in air. The $\mathrm{N}_{2}$ adsorption-desorption measurements was performed on a BELSORP-MINIII instrument at $77 \mathrm{~K}$ (BEL Japan). X-ray photoelectron spectroscopy (XPS) measurements of the powders were performed on VG ESCALAB220i-XL (Thermo Scientific) with the monochromatic Al X-ray source. 
2.3 Electrochemical measurements: The electrochemical properties were evaluated in $6 \mathrm{M} \mathrm{KOH}$ aqueous solution by using CHI-660D electrochemical workstation.

\subsubsection{Three-electrode system configuration}

The conventional three-electrode system consist of the working electrode, counter electrode ( $\mathrm{Pt}$ foil) and reference electrode $(\mathrm{Ag} / \mathrm{AgCl})$. The working electrode was prepared by pressing the mixed slurry (the $\mathrm{P}-\mathrm{NiO}$, carbon black and polyvinylidene fluoride with a mass ratio of 80:10:10 in N-methyl-2-pyrrolidone) on circular nickel foam $(\mathrm{d}=1.2 \mathrm{~cm})$ and dried at $90{ }^{\circ} \mathrm{C}$ overnight. The loading mass of $\mathrm{P}-\mathrm{NiO}$ was $5.131 \mathrm{mg} \mathrm{cm}^{-2}$.

The specific capacity $\left(\mathrm{mAh} \mathrm{g}^{-1}\right)$ is calculated from the cyclic voltammetry curves based on the equation of $Q=\frac{\int I d V}{2 v \mathrm{~m}}$, and from discharge curves according to the formula of $Q=\frac{I t}{m}$, while the specific capacitance $\left(\mathrm{F} \mathrm{g}^{-1}\right)$ is also calculated from the discharge curves using equation of $C=\frac{I \Delta t}{m \Delta V}$, where $v, m$ and $I$ stand for the scan rate, weight and discharge current density, respectively. $\Delta \mathrm{t}$ and $\Delta V$ refer to the discharge time and voltage window of P-NiO electrode, respectively.[41-43]

\subsubsection{Two-electrode system configuration}

For asymmetric device (named $\mathrm{P}-\mathrm{NiO} / / \mathrm{AC}$ ) fabricated by $\mathrm{P}-\mathrm{NiO}$ and activated carbon (AC), the cyclic voltammetry (CV) and galvanostatic charge/discharge (GCD) curves were tested by using CHI-660D electrochemical workstation. The cycling stability was measured in the Battery Testing System (Neware, CT-4008). The energy density $E\left(\mathrm{Wh} \mathrm{kg}^{-1}\right)$ and power density $P\left(\mathrm{~W} \mathrm{~kg}^{-1}\right)$ was calculated according to the following formulas: [44, 45] 


$$
\begin{aligned}
E & =\frac{I \int V d t}{\mathrm{M}} \\
P & =\frac{E}{t}
\end{aligned}
$$

where $I, V$ and $t$ represent the discharge current density, cell voltage and discharge time of the asymmetric device, respectively. $M$ is the total mass of two electrodes in the asymmetric device.

\section{Results and Discussion}

\subsection{Characterization of the prepared materials}

The synthetic schematic diagram of $\mathrm{P}-\mathrm{NiO}$ was shown in Figure 1, including the pretreatment of rape pollen grains, the chemical bath deposition and subsequent calcination. Obviously, the natural pollen grains have the elliptical morphology with many brain corrugation-like grooves on the surface (Figure S1a, Figure S1b). After pretreatment, part of core materials (proteins, amino acids, vitamins and nucleic acids, etc.) were removed, showing the hollow structure (Figure S1c). The porous structure (Figure S1e, Figure S1f) was obtained after calcination at $500{ }^{\circ} \mathrm{C}$.

\section{$<$ Figure 1 here $>$}

Besides, to analyze the decomposition temperature and thermal stability of as-prepared samples, TGA analysis were performed from room temperature to $650{ }^{\circ} \mathrm{C}$ in Figure S2. In the case of Pollen-B and precursor of P-NiO, the mass loss before 160 ${ }^{\circ} \mathrm{C}$ was due to the elimination of the adsorbed water and intercalated water, while the mass loss between $160{ }^{\circ} \mathrm{C}$ and $490{ }^{\circ} \mathrm{C}$ was ascribed to the decomposition of organic components.[44, 46] It is worth noting that no obvious mass loss is observed after 490 ${ }^{\circ} \mathrm{C}$, indicating the calcination of $500{ }^{\circ} \mathrm{C}$ is perfect enough to completely remove the 
pollen grains in $\mathrm{P}-\mathrm{NiO}$. Last but not least, the barely unobserved mass loss of $\mathrm{P}-\mathrm{NiO}$ in TGA curves indicating the high thermal stability of our sample.

Figure S3 shows the XRD patterns and SEM images (a-j) of as-prepared products calcinated at different temperatures (from $400{ }^{\circ} \mathrm{C}$ to $600{ }^{\circ} \mathrm{C}$ ). It can be observed that the rhombohedral $\mathrm{NiO}$ is completely formed at all the measured temperature, which indicates that our products may possess a high thermal stability. However, the elliptical morphology exhibits a remarkable collapse at high calcination temperature (especially at $600^{\circ} \mathrm{C}$ ), which is the main factor result in the worse electrochemical performances at $600^{\circ} \mathrm{C}$ (Figure S3 k-m). Obviously, the porous and hollow elliptical morphology constructed by abundant nanosheets can improve the diffusion of ions in electrolyte, facilitate the ion transfer and favors the enhanced electrochemical properties. Owing to the ideal morphology and outstanding electrochemical performance, we chose the products calcinated at $500{ }^{\circ} \mathrm{C}$ in this paper to illustrate the energy storage performance of pollen-tempalted $\mathrm{NiO}$.

SEM images of P-NiO before calcination show the elliptical structure with nanoplates on the surface (Figure S4). While, after calcination, the rhombohedral NiO (JCPDS 01-089-3080, Figure 2a) was obtained, indicating the addition of pollen template has no influence on the phase of P-NiO (Figure S1a, Figure S1d). The as-prepared $\mathrm{P}-\mathrm{NiO}$ with the porous and hollow elliptical microstructure self-assembled from nanosheets was obtained (Figure 2b).[47] The external surface was covered with numerous uniform nanosheets (5-20 nm in thickness, Figure 2c). Those thin and highly twisted nanosheets spontaneous connected with each other to form the porous elliptical 
structure (Figure 2d).[48] Furthermore, Figure 2e showed the SEM image observed from a destruction morphology of $\mathrm{P}-\mathrm{NiO}$, which revealed the hollow structure inside the P-NiO. The enlarged view in illustration clearly showed the porous microstructure in the smooth inner surface of P-NiO. Besides, the results of element composition demonstrate the atomic ratio of $\mathrm{Ni} / \mathrm{O}$ was being 1:1 (Figure $2 \mathrm{f}$ ), further demonstrating the successful preparation of $\mathrm{P}-\mathrm{NiO}$ by using rape pollen grains as template.

$<$ Figure 2 here>

As comparison, the SEM and TEM images of $\mathrm{W}-\mathrm{NiO}$ were also measured, which showed the microspheres assembled from abundant nanosheets (Figure S5), demonstrating the pollen grains play a vital role in obtaining $3 \mathrm{D}$ porous/hollow morphology. Furthermore, pollen-templated $\mathrm{SnO}_{2}$ and $\mathrm{TiO}_{2}$ with porous/hollow elliptical structures successful prepared (Figure S6), suggesting the generality and superiority of this strategy.

TEM measurement was performed to investigate the morphology and crystalline of as-prepared P-NiO (Figure 3). The bright-field microscopy image in Figure 3a reveals that $\mathrm{P}-\mathrm{NiO}$ was consisted of numerous nanosheets spontaneous forming porous and hollow microstructure. Figure $3 b$ clearly demonstrates the detailed images of nanosheets. The diffraction rings in the illustration indicating the polycrystalline nature of P-NiO. High-magnification TEM image show the lattice fringe with an interplanar spacing of $0.240 \mathrm{~nm}$, which is in good agreement with the $d$ value of (003) plane in rhombohedral NiO (Figure 3c). The TEM results are highly consistent with the SEM and XRD conclusions, further demonstrating the successful synthetize of porous and 
hollow P-NiO elliptical structure with a large number of nanosheets on the surface.

$<$ Figure 3 here $>$

Nitrogen adsorption-desorption analysis was performed to obtain the specific surface area and pore size distribution of as-prepared samples, as presented in Figure 4a. According to the IUPAC classification, both of P-NiO and W-NiO showed the type III adsorption-desorption isotherms with a distinct wide H3 type of hysteresis loop.[49] The Brunauere-Emmette-Teller (BET) specific surface area of P-NiO is $73.78 \mathrm{~m}^{2} \mathrm{~g}^{-1}$, higher than $\mathrm{W}-\mathrm{NiO}\left(64.13 \mathrm{~m}^{2} \mathrm{~g}^{-1}\right)$. The illustration in Figure $4 \mathrm{a}$ presented the Barret-Joyner-Halenda (BJH) pore size distribution calculated from desorption branch, both of $\mathrm{P}-\mathrm{NiO}$ and $\mathrm{W}-\mathrm{NiO}$ showed a wide mesopore range pore size distribution. The average pore size of $\mathrm{P}-\mathrm{NiO}$ is $7.883 \mathrm{~nm}$, higher than that of $\mathrm{W}-\mathrm{NiO}(3.411 \mathrm{~nm})$. In this regard, it can be expected that the different surface area together with the average pore size properties will lead to differences in ion transport and diffusion behavior during redox reactions, resulting in different electrochemical performance. [50] [51]

To determine the elemental composition of P-NiO, XPS measurements were performed as presented in Figure $4(\mathrm{~b}-\mathrm{d})$. The wide-range spectrum of $\mathrm{P}-\mathrm{NiO}$ indicating the existence of $\mathrm{Ni}, \mathrm{O}$ and $\mathrm{C}$ elements (Figure 4b). The existence of $\mathrm{C}$ elements are ascribed to air exposure. The high resolution spectra of $\mathrm{Ni} 2 \mathrm{p}$ can be fitted into a pair of main peak and satellite peak, as shown in Figure 4c. The binding energies located at 855.0 and $873.8 \mathrm{eV}$ corresponding to the Ni 2p3/2 and Ni 2p1/2, which are the typical Ni-O bonds in NiO.[52] The peaks centered at 861.4 and 880.1 $\mathrm{eV}$ are the shake-up satellite peaks (marked as "Sat.") ascribed to the Ni 2p3/2 and Ni 
2p1/2, respectively.[45] Besides, in the high resolution spectra of $\mathrm{O} 1 \mathrm{~s}$ (Figure 4d), the binding energy at 529.5 and $531.9 \mathrm{eV}$ was ascribed to the typical Ni-O bonds and $\mathrm{H}-\mathrm{O}-\mathrm{H}$ bond of residual water, respectively.[52, 53]

$<$ Figure 4 here>

\subsection{Electrochemical performance}

\subsubsection{Three-electrode system}

To evaluate the potential applications in supercapacitors, the electrochemical behaviors of P-NiO, W-NiO, Pollen-A, Pollen-C and Ni foam were investigated by $\mathrm{CV}$ technique at a scan rate of $10 \mathrm{mVs}^{-1}$. Obviously, the relative area surrounded by $\mathrm{CV}$ curve of $\mathrm{P}-\mathrm{NiO}$ is larger than the other electrodes, indicating the outstanding electrochemical energy storage performance (Figure 5a), which was also identified by the GCD curves in Figure 5b. The longer discharge process of $\mathrm{P}-\mathrm{NiO}$ indicating the higher specific capacitance compared with W-NiO. Furthermore, the GCD curves of $\mathrm{Ni}$ foam, Pollen-A and Pollen-C in the illustration of Figure $5 \mathrm{~b}$ exhibit shorter discharge process, the calculated smaller specific capacitance implying the negligible contribution in energy storage of these electrodes.

To further explore the electrochemical energy storage properties of $\mathrm{P}-\mathrm{NiO}$, a series of electrochemical tests were performed. The representative $\mathrm{CV}$ curves of $\mathrm{P}-\mathrm{NiO}$ at different scan rates are presented in Figure 5c. It is obvious that a pair of redox peaks can be observed in all scan rates. According to the reported literatures, the redox peaks is due to the reversible redox reaction between $\mathrm{NiO}$ and $\mathrm{NiOOH}$, as described in the following form:[54, 55] 


$$
\mathrm{NiO}+\mathrm{OH}^{-} \leftrightharpoons \mathrm{NiOOH}+e^{-}
$$

The P-NiO electrode achieves specific capacity of $141.0 \mathrm{mAh} \mathrm{g}^{-1}$ at $5 \mathrm{mVs}^{-1}$ and 87.0 $\mathrm{mAh} \mathrm{g}^{-1}$ at $20 \mathrm{mVs}^{-1}$. Furthermore, all the GCD curves of P-NiO exhibit symmetric charge/discharge processes and similar shapes even at a high current density of $30 \mathrm{~A} \mathrm{~g}^{-1}$, implying the excellent electrochemical reversibility and lower polarization of as-prepared electrode (Figure 5d).[56] The specific capacitance was calculated and plotted in Figure 5e. Promisingly, the P-NiO electrode exhibits high capacity of 198.7

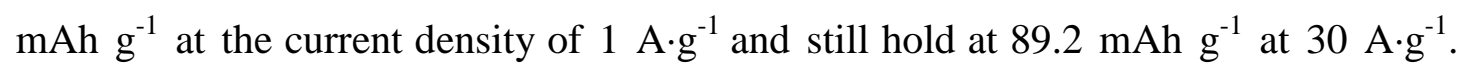
Besides, the corresponding specific capacitances are also calculated to be of $1589 \mathrm{~F} \mathrm{~g}^{-1}$ and $714 \mathrm{~F} \mathrm{~g}^{-1}$ at $1 \mathrm{~A} \mathrm{~g}^{-1}$ and $30 \mathrm{~A} \mathrm{~g}^{-1}$, respectively. The superior electrochemical performances are ascribed to the 3D porous/hollow elliptical microstructure with numerous nanosheets on the surface. This morphology has the unimpeded ion/electron diffusion channel and ubiquitous cavities, which can promote the diffusion and transfer of ions, improving the electrochemical performance. In addition, the specific capacitance decreases as the current density increases for the increment of voltage drop and insufficient active material involved in the redox reaction under high current densities.[57]

Furthermore, the good electrochemical properties of $\mathrm{P}-\mathrm{NiO}$ were further confirmed by the long cycling life (Figure 5f). During 5000 successive cycles at $10 \mathrm{~A}$ $\mathrm{g}^{-1}$, the specific capacitance value slowly decreased and retained $95.7 \%$ of its initial specific capacitance value. The detailed GCD curves was also plotted in the insert in Figure 5f. The similar GCD shapes indicating the high cycling performance of $\mathrm{P}-\mathrm{NiO}$, 
which may be attributed to the high chemical stability of this distinctive 3D elliptical microstructure. In addition, the electrochemical impedance spectroscopy (EIS) spectrum was measured at the open circuit potential within the frequency range from $100 \mathrm{kHz}$ to $0.01 \mathrm{~Hz}$ at amplitude of $5 \mathrm{mV}$ (Figure S7). The corresponding equivalent circuit was displayed in the illustration. The $\mathrm{x}$-intercept in high-frequency region is the internal resistance $\left(\mathrm{R}_{\mathrm{s}}, 0.69 \Omega\right)$, including the intrinsic resistance of $\mathrm{P}-\mathrm{NiO}$, the ionic resistance of electrolyte and contact resistance between current collector and electrode material.[58] The almost invisible semicircle corresponding to the charge transfer resistance $\left(\mathrm{R}_{\mathrm{ct}}\right)$, revealing the rapid and fast Faradaic reactions.[59] The line in the low-frequency region is described as Warburg resistance $\left(Z_{w}, 0.36 \Omega\right)$, which is the index of electrolyte diffusion/transport process.[60] $\mathrm{C}_{\mathrm{dl}}$ and $\mathrm{C}_{\mathrm{L}}$ are the double layer capacitance and the limit capacitance, respectively. The EIS data confirmed the higher electrical conductivity of $\mathrm{P}-\mathrm{NiO}$, the lower resistance in the electrochemical reactions, the easier ion diffusion and electrolyte penetration, which is beneficial to the improved electrochemical performance.

$<$ Figure 5 here >

The significant enhanced electrochemical performances are comparable with those nickel oxides electrode reported in literature, presented in Table S1.[61-71] The promising electrochemical performance of $\mathrm{P}-\mathrm{NiO}$ can be attributed to the following factors: (1) the porous and hollow 3D elliptical microstructure consisted of numerous nanosheets. This morphology has the unimpeded ion/electron diffusion channel and ubiquitous cavities, which can improve the diffusion of ions in electrolyte, facilitate the 
ion transfer inside the pore structure and provide more active sites for redox reaction, consequently enhance the electrochemical performance.[72-74] (2) The higher specific surface area with the pore size distribution in the mesopore range, providing the effective transport pathway between electrolyte and electrode materials, accelerating the diffusion of ions in electrolyte, and improving the efficient utilization of active materials. $[75,76]$

\subsubsection{Two-electrode system}

To evaluate the practical application, an asymmetric supercapacitor (ASC) was firstly fabricated by using $\mathrm{P}-\mathrm{NiO}$ and $\mathrm{AC}$ as anode and cathode, respectively, separated by a glass membrane. Firstly, the AC electrode was characterized as shown in Figure S8. Based on the charge balance theory, $[77,78]$ the mass ratio of $\mathrm{P}-\mathrm{NiO}$ and $\mathrm{AC}$ was calculated to be 0.55 (detailed information as seen in Supplementary Information). According to the individual potential window (Figure 6a), the theoretical maximum voltage of $\mathrm{P}-\mathrm{NiO} / / \mathrm{AC}$ device is $1.8 \mathrm{~V}$. To determine the suitable working voltage, the $\mathrm{CV}$ curves of the ASC under different voltage windows are exhibited in Figure $6 \mathrm{~b}$. The curled-up tail in the curves over $1.7 \mathrm{~V}$ implies the release of oxygen, which is pernicious for the packaged full cell in the practical applications.[79] On the other hand, the redox reaction is insufficient under 1.6 V. Therefore, the suitable voltage window of the device is determined to be $0-1.65 \mathrm{~V}$. A series of $\mathrm{CV}$ curves with different scan rates are shown in Figure 6c. The shapes of the CV profiles are still maintained even at a high scan rate of $100 \mathrm{mV} \mathrm{s}^{-1}$, illustrating the excellent capacitive behavior, fast ion transfer and low resistance of the asymmetric supercapacitor.[80] The GCD curves at different 
current densities are plotted in Figure 6d. The symmetrical charge/discharge curves and almost negligible voltage drop indicate the good electrochemical reversibility and small internal resistance of the asymmetric device.[41] The specific capacitance was calculated to vary from $128 \mathrm{Fg}^{-1}$ at $1 \mathrm{~A} \mathrm{~g}^{-1}$ to $69 \mathrm{~F} \mathrm{~g}^{-1}$ at $20 \mathrm{~A} \mathrm{~g}^{-1}$.

As the crucial factors to evaluate supercapacitors, the energy/power density of $\mathrm{P}-\mathrm{NiO} / / \mathrm{AC}$ device were calculated and shown in Figure 6e. The asymmetric device reveals a high energy density of $66.4 \mathrm{Wh} \mathrm{kg}^{-1}$ at $1136.6 \mathrm{~W} \mathrm{~kg}^{-1}$ and a high power density of $22.0 \mathrm{~kW} \mathrm{~kg}$ at $34.5 \mathrm{Wh} \mathrm{kg}^{-1}$, suggesting the promising applications in energy storage.[44,53,62,65,67,70,83] Considering the calculation of energy/power density is highly depending on the whole electrode mass, it still has much room for the improvement of energy/power density by employing other cathode electrode materials with high electrochemical performance.

Furthermore, the cycling stability and coulombic efficiency of P-NiO//AC asymmetric device were measured at $8 \mathrm{~A} \mathrm{~g}^{-1}$ (Figure 6f.). The specific capacitances increased in the first 4000 cycles for the activation of samples and then became constant after the subsequent 6000 cycles (the detailed GCD curves plotted in the insert in Figure 6f). Promising, the specific capacitance retention is as high as $103.3 \%$ after 10000 cycles, suggesting the superior cycling stability and potential application of the asymmetric device. Except for the excellent cycling stability of P-NiO itself, the superior stability can also be ascribed to the intercalation of AC.[81] In addition, the device exhibits a high coulombic efficiency of $98.5 \%$, demonstrating the high conversion efficiency of this device.[82] As discussed above, the P-NiO//AC 
asymmetric device demonstrates excellent electrochemical properties, including high specific capacitance, high power/energy density and excellent cycling ability. The high electrochemical performance of the asymmetric device is attributed to the porous and hollow 3D network-like $\mathrm{P}-\mathrm{NiO}$ elliptical microstructures self-assembled from abundant nanosheets, high specific surface area with mesopore range pore size distribution and wide voltage window.

$<$ Figure 6 here >

\section{Conclusions}

In summary, porous and hollow pollen-templated $\mathrm{NiO}$ elliptical microstructures were successfully synthesized via a simple and efficient chemical bath deposition. Benefiting from the unique morphology and high specific surface area, the pollen-templated $\mathrm{NiO}$ exhibit high specific capacity (198.7 $\mathrm{mAh} \mathrm{g}^{-1}$ at $\left.1 \mathrm{~A} \cdot \mathrm{g}^{-1}\right)$, excellent rate capability $\left(89.2 \mathrm{mAh} \mathrm{g}^{-1}\right.$ at $\left.30 \mathrm{~A} \cdot \mathrm{g}^{-1}\right)$ and long cycling life. Furthermore, the pollen-templated $\mathrm{NiO} / / \mathrm{AC}$ asymmetric supercapacitor shows excellent cycling stability (103.3\% retention after 10000 cycles) and high energy/power density (a high energy density of $66.4 \mathrm{Wh} \mathrm{kg}^{-1}$ and power density of $22.0 \mathrm{~kW} \mathrm{~kg}^{-1}$ ). Moreover, this work also provides an efficient process for synthesizing porous hollow transition metal oxides.

\section{Acknowledgements}

This work was supported by the National Science Foundation of China (NSFC No. 51372195), the CSS project (Grant No. YK2015-0602006), the Ministry of Science and Technology of China through a 973-Project (No. 2012CB619401), the 
Fundamental Research Funds for the Central Universities (2013JDGZ03), and Program for Innovative Research Team in University of Ministry of Education of China (IRT13034). X.J. Lou would like to thank the "One Thousand Youth Talents" program for support. The author would like to thank Chuansheng Ma (International Center for Dielectric Research, Xi'an Jiaotong University) for the TEM Testing. 


\section{References}

[1] J. Wang, B. Ding, X. Hao, Y. Xu, Y. Wang, L. Shen, H. Dou, X. Zhang, A modified molten-salt method to prepare graphene electrode with high capacitance and low self-discharge rate, Carbon 102 (2016) 255-261.

[2] A. Yu, Z. Chen, R. Maric, L. Zhang, J. Zhang, J. Yan, Electrochemical supercapacitors for energy storage and delivery: Advanced materials, technologies and applications, Applied Energy 153 (2015) 1-2.

[3] M. Wagemaker, F.M. Mulder, Properties and Promises of Nanosized Insertion Materials for Li-Ion Batteries, Acc. Chem. Res. 46 (2013) 1206-1215.

[4] P. Simon, Y. Gogotsi, Materials for electrochemical capacitors, Nature Mater. 7 (2008) 845-854.

[5] C. Qu, Y. Jiao, B. Zhao, D. Chen, R. Zou, K.S. Walton, M. Liu, Nickel-based pillared MOFs for high-performance supercapacitors: Design, synthesis and stability study, Nano Energy (2016) 66-73.

[6] G. Zhang, H. Liu, J. Qu, J. Li, Two-dimensional layered $\mathrm{MoS}_{2}$ : rational design, properties and electrochemical applications, Energy Environ. Sci. 9 (2016) 1190-1209.

[7] B. Chang, H. Yin, X. Zhang, S. Zhang, B. Yang, Chemical blowing strategy synthesis of nitrogen-rich porous graphitized carbon nanosheets: Morphology, pore structure and supercapacitor application, Chem. Eng. J. 312 (2017) 191-203.

[8] S. Faraji, F.N. Ani, Microwave-assisted synthesis of metal oxide/hydroxide composite electrodes for high power supercapacitors-A review, J. Power Sources 263 
(2014) 338-360.

[9] K. Krishnamoorthy, G.K. Veerasubramani, S. Radhakrishnan, S.J. Kim, One pot hydrothermal growth of hierarchical nanostructured $\mathrm{Ni}_{3} \mathrm{~S}_{2}$ on $\mathrm{Ni}$ foam for supercapacitor application, Chem. Eng. J. 251 (2014) 116-122.

[10] B. Li, M. Zheng, H. Xue, H. Pang, High performance electrochemical capacitor materials focusing on nickel based materials, Inorg. Chem. Front. 3 (2016) 175-202.

[11] N. A. Samsudin, Z. Zainal, H. N. Lim, Y. Sulaiman,S. K. Chang, Titania Nanotubes Synthesised via the Electrochemical Anodisation Method: Synthesis and Supercapacitor Applications, Pertanika Journal of Scholarly Research Reviews (2016) $107-128$.

[12] M. Vangari, T. Pryor, L. Jiang, Supercapacitors: Review of materials and fabrication methods, J. Energy Eng. 139 (2012) 72-79.

[13] B. Dong, X. Zhang, X. Xu, G. Gao, S. Ding, J. Li, B. Li, Preparation of scale-like nickel cobaltite nanosheets assembled on nitrogen-doped reduced graphene oxide for high-performance supercapacitors, Carbon 80 (2014) 222-228.

[14] M.S. Kolathodi, M. Palei, T.S. Natarajan, Electrospun NiO nanofibers as cathode materials for high performance asymmetric supercapacitors, J. Mater. Chem. A 3 (2015) 7513-7522.

[15] L. Feng, Y. Zhu, H. Ding, C. Ni, Recent progress in nickel based materials for high performance pseudocapacitor electrodes, J. Power Sources 267 (2014) 430-444.

[16] G.Q. Zhang, L. Yu, H.E. Hoster, X.W. Lou, Synthesis of one-dimensional hierarchical NiO hollow nanostructures with enhanced supercapacitive performance, 
Nanoscale 5 (2013) 877-881.

[17] Q. Lu, J.G. Chen, J.Q. Xiao, Nanostructured Electrodes for High-Performance Pseudocapacitors, Angew. Chem. Int. Ed. 52 (2013) 1882-1889.

[18] B. Li, M. Zheng, H. Xue, H. Pang, High performance electrochemical capacitor materials focusing on nickel based materials, Inorg. Chem. Front. 3 (2016) 175-202.

[19] A. Paravannoor, S.V. Nair, P. Pattathil, M. Manca, A. Balakrishnan, High voltage supercapacitors based on carbon-grafted $\mathrm{NiO}$ nanowires interfaced with an aprotic ionic liquid, Chem. Commun. 51 (2015) 6092-6095.

[20] G. Cheng, W. Yang, C. Dong, T. Kou, Q. Bai, H. Wang, Z. Zhang, Ultrathin mesoporous $\mathrm{NiO}$ nanosheet-anchored 3D nickel foam as an advanced electrode for supercapacitors, J. Mater. Chem. A 3 (2015) 17469-17478.

[21] X. Zheng, H. Wang, C. Wang, Z. Deng, L. Chen, Y. Li, T. Hasan, B.L. Su, 3D interconnected macro-mesoporous electrode with self-assembled $\mathrm{NiO}$ nanodots for high-performance supercapacitor-like Li-ion battery, Nano Energy 22 (2016) 269-277.

[22] H. Xiao, F. Qu, X. Wu, Ultrathin $\mathrm{NiO}$ nanoflakes electrode materials for supercapacitors, Appl. Surf. Sci. 360 (2016) 8-13.

[23] J. Min, J. Liu, M. Lei, W. Wang, Y. Lu, L. Yang, Q. Yang, G. Liu, N. Su, Self-Assembly of Parallelly Aligned NiO Hierarchical Nanostructures with Ultrathin Nanosheet Subunits for Electrochemical Supercapacitor Applications, ACS Appl. Mater. Interfaces 8 (2016) 780-791.

[24] A. Zhou, X. Dai, Y. Lu, Q. Wang, M. Fu, J. Li, Enhanced Interfacial Kinetics 
and High-Voltage/High-Rate Performance of $\mathrm{LiCoO}_{2}$ Cathode by Controlled Sputter-Coating with a Nanoscale $\mathrm{Li}_{4} \mathrm{Ti}_{5} \mathrm{O}_{12}$ Ionic Conductor, ACS Appl. Mater. Interfaces 8 (2016) 34123-34131.

[25] L. Yu, L. Zhang, H.B. Wu, X.W.D. Lou, Formation of $\mathrm{Ni}_{\mathrm{x}} \mathrm{Co}_{3-\mathrm{x}} \mathrm{S}_{4}$ hollow nanoprisms with enhanced pseudocapacitive properties, Angew. Chem. Int. Ed. 53 (2014) $3711-3714$

[26] W. Ji, J. Ji, X. Cui, J. Chen, D. Liu, H. Deng, Q. Fu, Polypyrrole encapsulation on flower-like porous $\mathrm{NiO}$ for advanced high-performance supercapacitors, Chem. Commun. 51 (2015) 7669-7672.

[27] C. Yan, F. Rosei, Hollow micro/nanostructured materials prepared by ion exchange synthesis and their potential applications, New J. Chem. 38 (2014) 1883-1904.

[28] T. Zhu, Z. Wang, S. Ding, J.S. Chen, X.W. Lou, Hierarchical nickel sulfide hollow spheres for high performance supercapacitors, RSC Adv. 1 (2011) 397-400.

[29] Y.G. Wang, Y.Y. Xia, Electrochemical capacitance characterization of NiO with ordered mesoporous structure synthesized by template SBA-15, Electrochim. Acta 51 (2006) 3223-3227.

[30] G. Zan, Q. Wu, Biomimetic and Bioinspired Synthesis of Nanomaterials/Nanostructures, Adv. Mater. 28 (2016) 2099-2147.

[31] Z. Ren, P.X. Gao, A review of helical nanostructures: growth theories, synthesis strategies and properties, Nanoscale 6 (2014) 9366-9400.

[32] Y. Fang, J.D. Berrigan, Y. Cai, S.R. Marder, K.H. Sandhage, Syntheses of 
nanostructured $\mathrm{Cu}$-and Ni-based micro-assemblies with selectable 3-D hierarchical biogenic morphologies, J. Mater. Chem. 22 (2012) 1305-1312.

[33] M.R. Weatherspoon, Y. Cai, M. Crne, M. Srinivasarao, K.H. Sandhage, 3D Rutile Titania-Based Structures with Morpho Butterfly Wing Scale Morphologies, Angew. Chem. Int. Ed. 47 (2008) 7921-7923.

[34] C. Zhang, J. Wang, R. Hu, Q. Qiao, X. Li, Synthesis and gas sensing properties of porous hierarchical $\mathrm{SnO}_{2}$ by grapefruit exocarp biotemplate, Sens. Actuators B: Chemical 222 (2016) 1134-1143.

[35] Y. Gao, L. Li, Y. Jin, Y. Wang, C. Yuan, Y. Wei, G. Chen, J. Ge, H. Lu, Porous carbon made from rice husk as electrode material for electrochemical double layer capacitor, Applied Energy 153 (2015) 41-47.

[36] X. Tao, J. Du, Y. Yang, Y. Li, Y. Xia, Y. Gan, H. Huang, W. Zhang, X. Li, TiC Nanorods Derived from Cotton Fibers: Chloride-Assisted VLS Growth, Structure, and Mechanical Properties, Cryst. Growth Des. 11 (2011) 4422-4426.

[37] Y. Lv, L. Gan, M. Liu, W. Xiong, Z. Xu, D. Zhu, D.S. Wright, A self-template synthesis of hierarchical porous carbon foams based on banana peel for supercapacitor electrodes, J. Power Sources 209 (2012) 152-157.

[38] Y. Zhao, M. Wei, J. Lu, Z.L. Wang, X. Duan, Biotemplated hierarchical nanostructure of layered double hydroxides with improved photocatalysis performance, ACS nano 3 (2009) 4009-4016.

[39] X. Tao, W. Chai, F. Xu, J. Luo, H. Xiao, C. Liang, Y. Gan, H. Huang, Y. Xia, W. Zhang, Bio-templated Fabrication of Highly Defective Carbon Anchored MnO Anode 
Materials with High Reversible Capacity, Electrochim. Acta 169 (2015) 159-167.

[40] Y. Xia, W. Zhang, Z. Xiao, H. Huang, H. Zeng, X. Chen, F. Chen, Y. Gan, X. Tao, Biotemplated fabrication of hierarchically porous $\mathrm{NiO} / \mathrm{C}$ composite from lotus pollen grains for lithium-ion batteries, J. Mater. Chem. 22 (2012) 9209-9215.

[41] Z. Fan, J. Yan, T. Wei, L. Zhi, G. Ning, T. Li, F. Wei, Asymmetric Supercapacitors Based on Graphene/ $\mathrm{MnO}_{2}$ and Activated Carbon Nanofiber Electrodes with High Power and Energy Density, Adv. Funct. Mater. 21 (2011) 2366-2375.

[42] T. Brousse, D. Belanger, J.W. Long, To Be or Not To Be Pseudocapacitive?, J. Electrochem. Soc. 162 (2015) A5185-A5189.

[43] B. Wang, J.S. Chen, Z. Wang, S. Madhavi, X.W. Lou, Green Synthesis of NiO Nanobelts with Exceptional Pseudo-Capacitive Properties, Adv. Energy Mater. 2 (2012) 1188-1192.

[44] W. Xu, B. Mu, A. Wang, Facile fabrication of well-defined microtubular carbonized kapok fiber/ $\mathrm{NiO}$ composites as electrode material for supercapacitor, Electrochim. Acta 194 (2016) 84-94.

[45] H. Lai, Q. Wu, J. Zhao, L. Shang, H. Li, R. Che, Z. Lyu, J. Xiong, L. Yang, X. Wang, Z. Hu, Mesostructured $\mathrm{NiO} / \mathrm{Ni}$ composites for high-performance electrochemical energy storage, Energy Environ. Sci. 9 (2016) 2053-2060.

[46] H. Li, B. Wang, X. He, J. Xiao, H. Zhang, Q. Liu, J. Liu, J. Wang, L. Liu, P. Wang, Composite of hierarchical interpenetrating 3D hollow carbon skeleton from lotus pollen and hexagonal $\mathrm{MnO}_{2}$ nanosheets for high-performance supercapacitors, $\mathrm{J}$. 
Mater. Chem. A 3 (2015) 9754-9762.

[47] H. Fang, L. Zhao, W. Yue, Y. Wang, Y. Jiang, Y. Zhang, Facile and large-scale preparation of sandwich-structured graphene-metal oxide composites as anode materials for Li-ion batteries, Electrochim. Acta 186 (2015) 397-403.

[48] J.W. Lee, T. Ahn, J.H. Kim, J.M. Ko, J.D. Kim, Nanosheets based mesoporous $\mathrm{NiO}$ microspherical structures via facile and template-free method for high performance supercapacitors, Electrochim. Acta 56 (2011) 4849-4857.

[49] S.H. Bae, J.E. Kim, H. Randriamahazaka, S.Y. Moon, J.Y. Park, I.K. Oh, Seamlessly Conductive 3D Nanoarchitecture of Core-Shell Ni-Co Nanowire Network for Highly Efficient Oxygen Evolution, Adv. Energy Mater. 7 (2017) 1601492.

[50] S. Ding, T. Zhu, J.S. Chen, Z. Wang, C. Yuan, X.W. Lou, Controlled synthesis of hierarchical $\mathrm{NiO}$ nanosheet hollow spheres with enhanced supercapacitive performance, J. Mater. Chem. 21 (2011) 6602-6606.

[51] Y. Yang, Y. Liang, Z. Zhang, Y. Zhang, H. Wu, Z. Hu, Morphology well-controlled synthesis of $\mathrm{NiO}$ by solvothermal reaction time and their morphology-dependent pseudocapacitive performances, J. Alloys Compd. 658 (2016) 621-628.

[52] C. Zhang, L. Qian, K. Zhang, S. Yuan, J. Xiao, S. Wang, Hierarchical porous $\mathrm{Ni} / \mathrm{NiO}$ core-shell with superior conductivity for electrochemical pseudo-capacitor and glucose sensor, J. Mater. Chem. A 3 (2015) 10519-10525.

[53] V. Senthilkumar, F.B. Kadumudi, N.T. Ho, J.W. Kim, S. Park, J.S. Bae, W.M. Choi, S. Cho, Y.S. Kim, NiO nanoarrays of a few atoms thickness on 3D nickel 
network for enhanced pseudocapacitive electrode applications, J. Power Sources 303 (2016) 363-371.

[54] J. Li, W. Zhao, F. Huang, A. Manivannan, N. Wu, Single-crystalline Ni(OH) 2 and $\mathrm{NiO}$ nanoplatelet arrays as supercapacitor electrodes, Nanoscale 3 (2011) 5103-5109.

[55] X. Zhang, W. Shi, J. Zhu, W. Zhao, J. Ma, S. Mhaisalkar, T.L. Maria, Y. Yang, H. Zhang, H.H. Hng, Q. Yan, Synthesis of porous NiO nanocrystals with controllable surface area and their application as supercapacitor electrodes, Nano Res. 3 (2010) 643-652.

[56] C. Yuan, J. Li, L. Hou, X. Zhang, L. Shen, X.W. Lou, Ultrathin mesoporous $\mathrm{NiCo}_{2} \mathrm{O}_{4}$ nanosheets supported on $\mathrm{Ni}$ foam as advanced electrodes for supercapacitors, Adv. Funct. Mater. 22 (2012) 4592-4597.

[57] H. Li, Y. Gao, C. Wang, G. Yang, A Simple Electrochemical Route to Access Amorphous Mixed-Metal Hydroxides for Supercapacitor Electrode Materials, Adv. Energy Mater. 5 (2015) 1401767.

[58] M. Liu, M. Shi, W. Lu, D. Zhu, L. Li, L. Gan, Core-shell reduced graphene oxide/MnOx@carbon hollow nanospheres for high performance supercapacitor electrodes, Chem. Eng. J. 313 (2017) 518-526.

[59] L. Wei, K. Tian, Y. Jin, X. Zhang, X. Guo, Three-dimensional porous hollow microspheres of activated carbon for high-performance electrical double-layer capacitors, Micropor. Mesopor. Mat. 227 (2016) 210-218.

[60] M. Liu, X. Wang, D. Zhu, L. Li, H. Duan, Z. Xu, Z. Wang, L. Gan, 
Encapsulation of $\mathrm{NiO}$ nanoparticles in mesoporous carbon nanospheres for advanced energy storage, Chem. Eng. J. 308 (2017) 240-247.

[61] M. Zhang, Q. Li, D. Fang, I.A. Ayhan, Y. Zhou, L. Dong, C. Xiong, Q. Wang, $\mathrm{NiO}$ hierarchical hollow nanofibers as high-performance supercapacitor electrodes, RSC Adv. 5 (2015) 96205-96212.

[62] F. Yu, L. Zhu, T. You, F. Wang, Z. Wen, Preparation of chestnut-like porous $\mathrm{NiO}$ nanospheres as electrodes for supercapacitors, RSC Adv. 5 (2015) 96165-96169.

[63] G. Cai, X. Wang, M. Cui, P. Darmawan, J. Wang, A.L.-S. Eh, P.S. Lee, Electrochromo-supercapacitor based on direct growth of $\mathrm{NiO}$ nanoparticles, Nano Energy 12 (2015) 258-267.

[64] Y. Zhang, J. Wang, H. Wei, J. Hao, J. Mu, P. Cao, J. Wang, S. Zhao, Hydrothermal synthesis of hierarchical mesoporous $\mathrm{NiO}$ nanourchins and their supercapacitor application, Mater. Lett. 162 (2016) 67-70.

[65] H. Yi, H. Wang, Y. Jing, T. Peng, X. Wang, Asymmetric supercapacitors based on carbon nanotubes@NiO ultrathin nanosheets core-shell composites and MOF-derived porous carbon polyhedrons with super-long cycle life, J. Power Sources 285 (2015) 281-290.

[66] Z. Yang, F. Xu, W. Zhang, Z. Mei, B. Pei, X. Zhu, Controllable preparation of multishelled $\mathrm{NiO}$ hollow nanospheres via layer-by-layer self-assembly for supercapacitor application, J. Power Sources 246 (2014) 24-31.

[67] M. Jing, C. Wang, H. Hou, Z. Wu, Y. Zhu, Y. Yang, X. Jia, Y. Zhang, X. Ji, Ultrafine nickel oxide quantum dots enbedded with few-layer exfoliative graphene for 
an asymmetric supercapacitor: Enhanced capacitances by alternating voltage, J. Power Sources 298 (2015) 241-248.

[68] M. Yao, Z. Hu, Z. Xu, Y. Liu, P. Liu, Q. Zhang, Template synthesis and characterization of nanostructured hierarchical mesoporous ribbon-like $\mathrm{NiO}$ as high performance electrode material for supercapacitor, Electrochim. Acta 158 (2015) 96-104.

[69] C. Wu, S. Deng, H. Wang, Y. Sun, J. Liu, H. Yan, Preparation of novel three-dimensional $\mathrm{NiO} /$ ultrathin derived graphene hybrid for supercapacitor applications, ACS Appl. Mater. Interfaces 6 (2014) 1106-1112.

[70] X. Ren, C. Guo, L. Xu, T. Li, L. Hou, Y. Wei, Facile Synthesis of Hierarchical Mesoporous Honeycomb-like NiO for Aqueous Asymmetric Supercapacitors, ACS Appl. Mater. Interfaces 7 (2015) 19930-19940.

[71] K.K. Purushothaman, I.M. Babu, B. Sethuraman, G. Muralidharan, Nanosheet-assembled $\mathrm{NiO}$ microstructures for high-performance supercapacitors, ACS Appl. Mater. Interfaces 5 (2013) 10767-10773.

[72] Z. Fan, J. Liang, W. Yu, S. Ding, S. Cheng, G. Yang, Y. Wang, Y. Xi, K. Xi, R.V. Kumar, Ultrathin NiO nanosheets anchored on a highly ordered nanostructured carbon as an enhanced anode material for lithium ion batteries, Nano Energy 16 (2015) $152-162$.

[73] S. Chabi, C. Peng, D. Hu, Y. Zhu, Ideal Three-Dimensional Electrode Structures for Electrochemical Energy Storage, Adv. Mater. 26 (2014) 2440-2445.

[74] J. Yang, Z. Ma, W. Gao, M. Wei, Layered Structural Co-Based MOF with 
Conductive Network Frames as a New Supercapacitor Electrode, Chem. Eur. J. 23 (2017) 631-636.

[75] X.J. Ma, L.B. Kong, W.B. Zhang, M.C. Liu, Y.C. Luo, L. Kang, Design and synthesis of 3D $\mathrm{Co}_{3} \mathrm{O}_{4} @ \mathrm{MMoO}_{4}(\mathrm{M}=\mathrm{Ni}, \mathrm{Co})$ nanocomposites as high-performance supercapacitor electrodes, Electrochim. Acta 130 (2014) 660-669.

[76] H. Sim, C. Jo, T. Yu, E. Lim, S. Yoon, J.H. Lee, J. Yoo, J. Lee, B. Lim, Reverse micelle synthesis of colloidal nickel-manganese layered double hydroxide nanosheets and their pseudocapacitive properties, Chem. Eur. J. 20 (2014) 14880-14884.

[77] V. Khomenko, E. R. Pinero, F. Béguin, Optimisation of an asymmetric manganese oxide/activated carbon capacitor working at $2 \mathrm{~V}$ in aqueous medium, $\mathrm{J}$. Power Sources 153 (2006) 183-190.

[78] V. Khomenko, E.R. Piñero, F. Béguin, High-energy density graphite/AC capacitor in organic electrolyte, J. Power Sources 177 (2008) 643-651.

[79] D. Li, Y. Gong, Y. Zhang, C. Luo, W. Li, Q. Fu, C. Pan, Facile Synthesis of Carbon Nanosphere/ $\mathrm{NiCo}_{2} \mathrm{O}_{4}$ Core-shell Sub-microspheres for High Performance Supercapacitor, Sci. Rep. 5 (2015) 12903

[80] X. Lu, D. Wu, R. Li, Q. Li, S. Ye, Y. Tong, G. Li, Hierarchical $\mathrm{NiCo}_{2} \mathrm{O}_{4}$ nanosheets@hollow microrod arrays for high-performance asymmetric supercapacitors, J. Mater. Chem. A 2 (2014) 4706-4713.

[81] J. Yan, Z. Fan, W. Sun, G. Ning, T. Wei, Q. Zhang, R. Zhang, L. Zhi, F. Wei, Advanced Asymmetric Supercapacitors Based on $\mathrm{Ni}(\mathrm{OH})_{2} /$ Graphene and Porous Graphene Electrodes with High Energy Density, Adv. Funct. Mater. 22 (2012) 
2632-2641.

[82] S. Wang, W. Li, L. Xin, M. Wu, X. Lou, High-performance nickel cobalt sulfide materials via low-cost preparation for advanced asymmetric supercapacitors, RSC Adv. 6 (2016) 42633-42642.

[83] Z. Wen, F. Yu, T. You, L. Zhu, L. Zhang, Y. Wu, A core-shell structured nanocomposite of $\mathrm{NiO}$ with carbon nanotubes as positive electrode material of high capacitance for supercapacitors, Mater. Res. Bull. 74 (2016) 241-247. 


\section{Figure captions}

Figure1 Fabrication scheme of as-prepared P-NiO. (a) rape pollen grains without treatment; (b) pretreated rape pollen grains; (c) $\mathrm{P}$-NiO.

Figure2 XRD and SEM images of P-NiO. (a) XRD patterns; (b) low-magnification SEM image; (c,d) high-magnifcation SEM image; (e) internal surface of $\mathrm{P}-\mathrm{NiO} ;(f)$ EDS spectrum.

Figure3 TEM images of P-NiO. (a,b) TEM ;(c) HRTEM.

Figure4 (a)Nitrogen adsorption-desorption isotherm of $\mathrm{P}$-NiO and $\mathrm{W}$-NiO (with the pore size distribution in the inset); XPS spectrum of (b)survey spectrum;(c) Ni $2 p$ spectrum and (d) $O$ 1s spectrum.

Figure 5 Electrochemical evaluations. (a) CV curves measured at $10 \mathrm{mV} \mathrm{s}^{-1}$; (b) GCD curves at $10 \mathrm{~A} \mathrm{~g}^{-1}$ (inset is the GCD curves of Ni foam, Pollen-A and Pollen-C at $1 \mathrm{~A}$ $\left.g^{-1}\right) ;(c) C V$ curves of $P$-NiO; (d) GCD curves; (e) rate capability performance; $(f)$ long-term cycling performance (inset is the GCD curves of 1 st,1000 th and 5000 th). Figure 6 Electrochemical evaluation of P-NiO//AC asymmetric supercapacitor. (a) $C V$ curves measured in three-electrode system; (b) CV curves under different voltages at $10 \mathrm{mV} \mathrm{s}^{-1}$; (c) CV curves under different scan rates; (d) GCD curves; (e) Ragone plot; $(f)$ cycling performance and coulombic efficiency (inset is the GCD curves of 1 st,4000 th and 10000 th). 


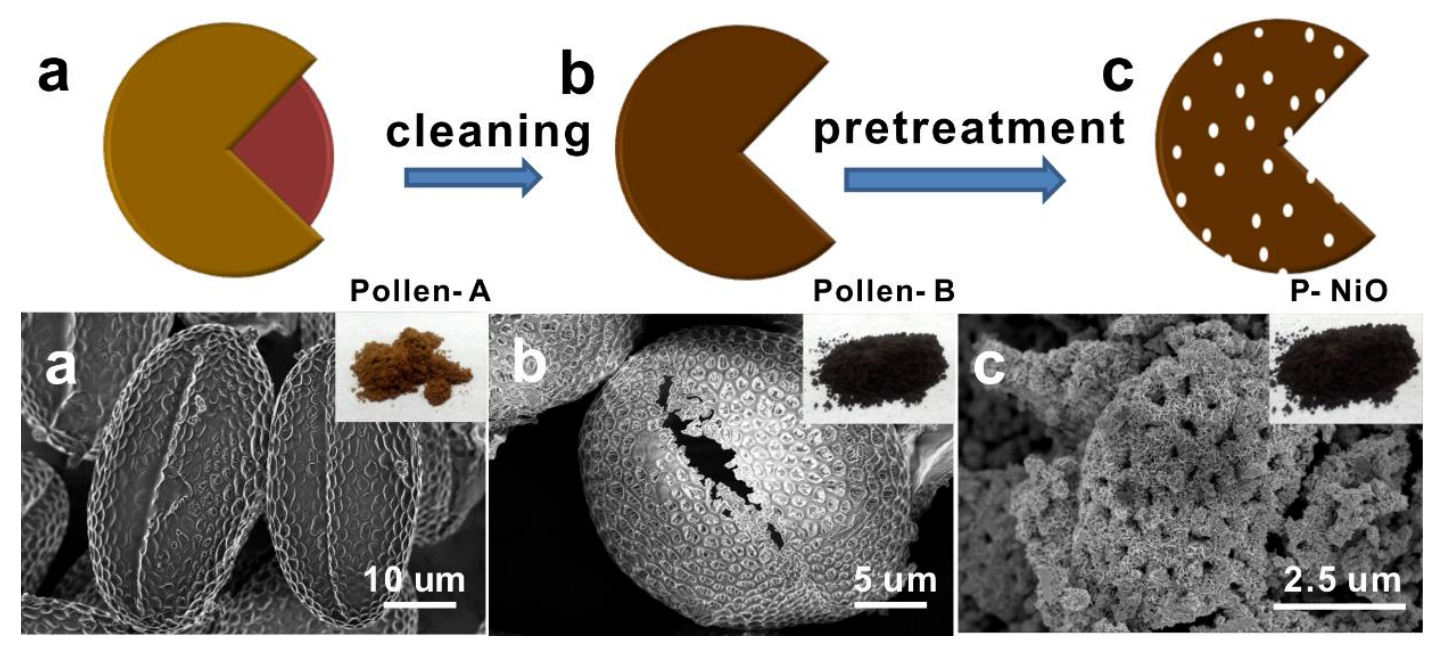

Figure 1 

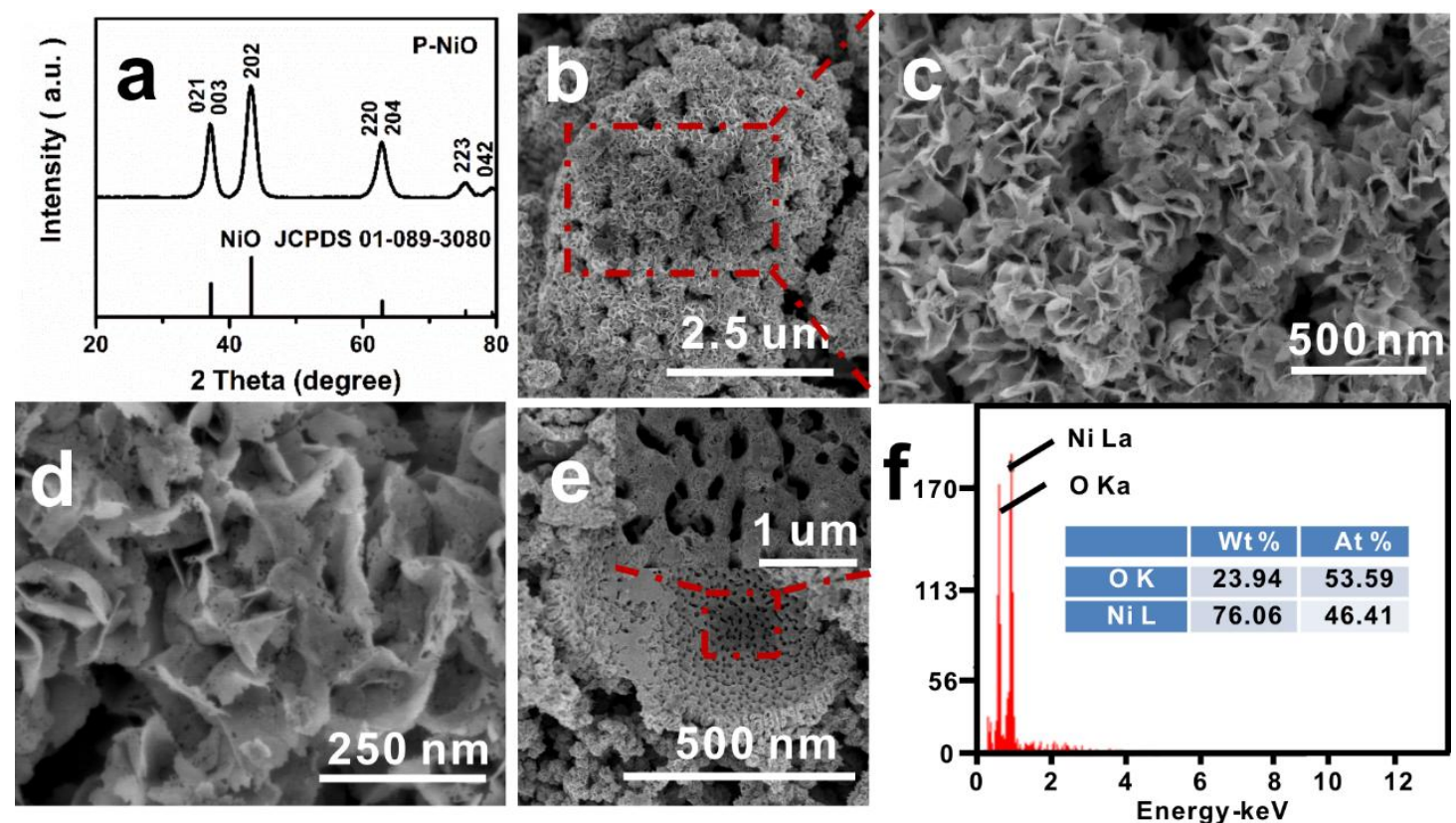

Figure 2 


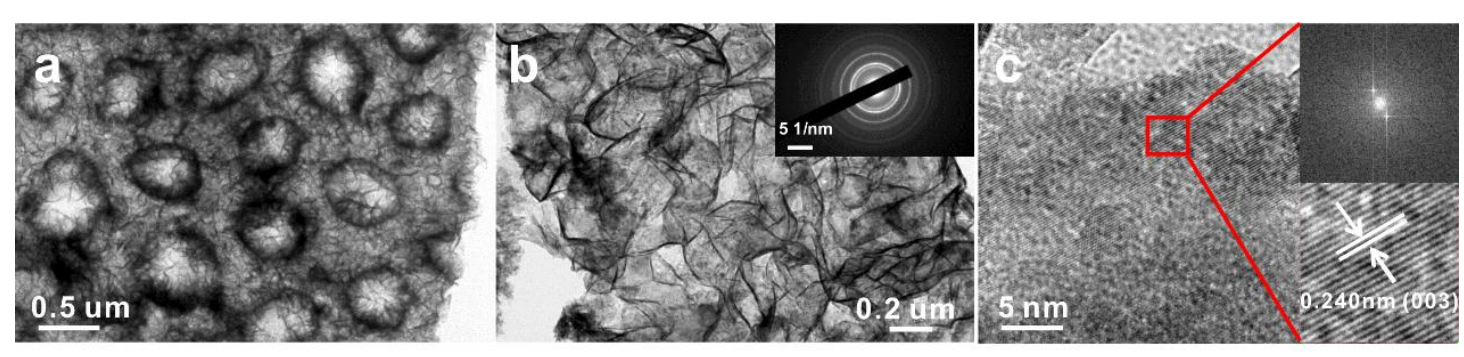

Figure 3 

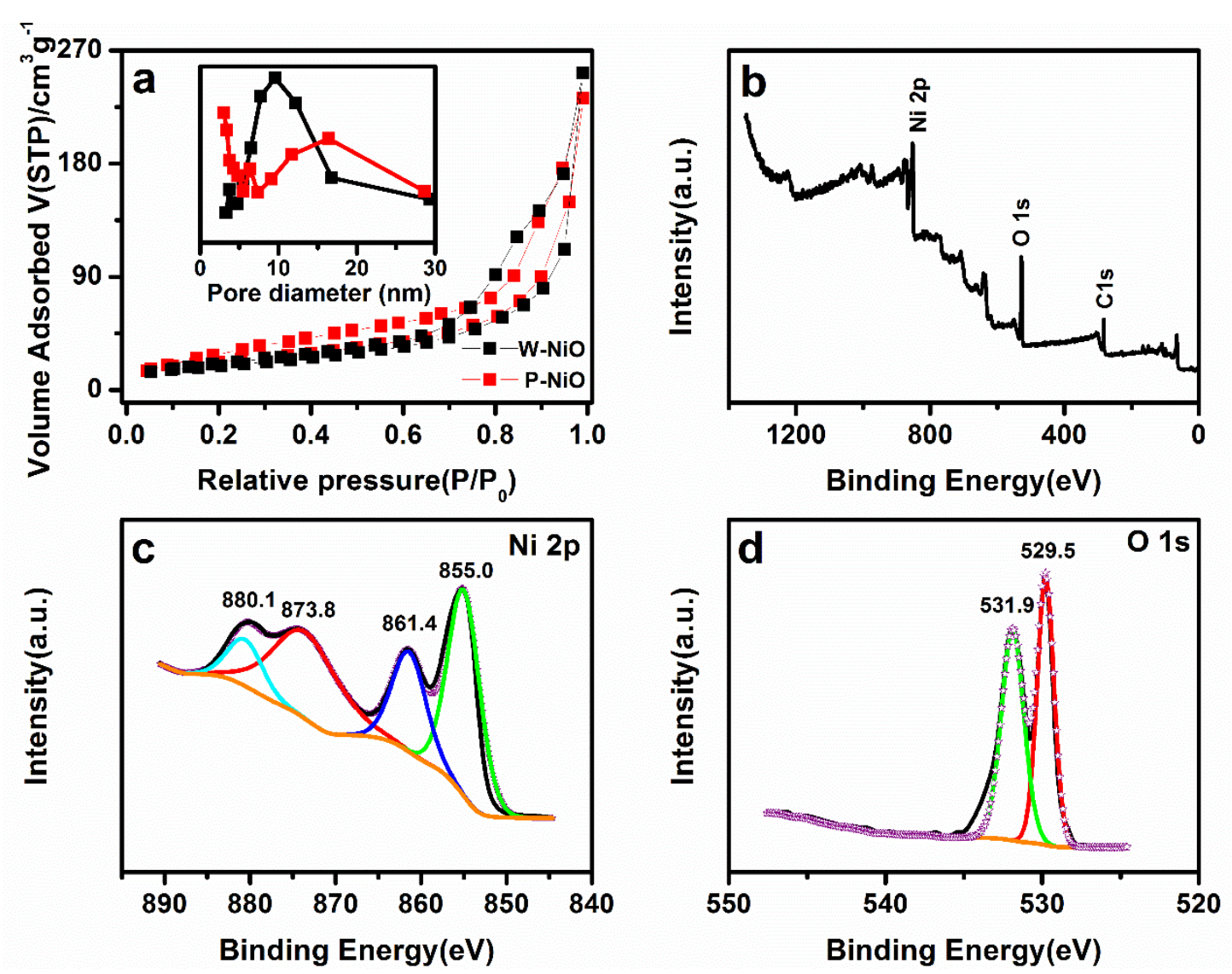

Figure 4 

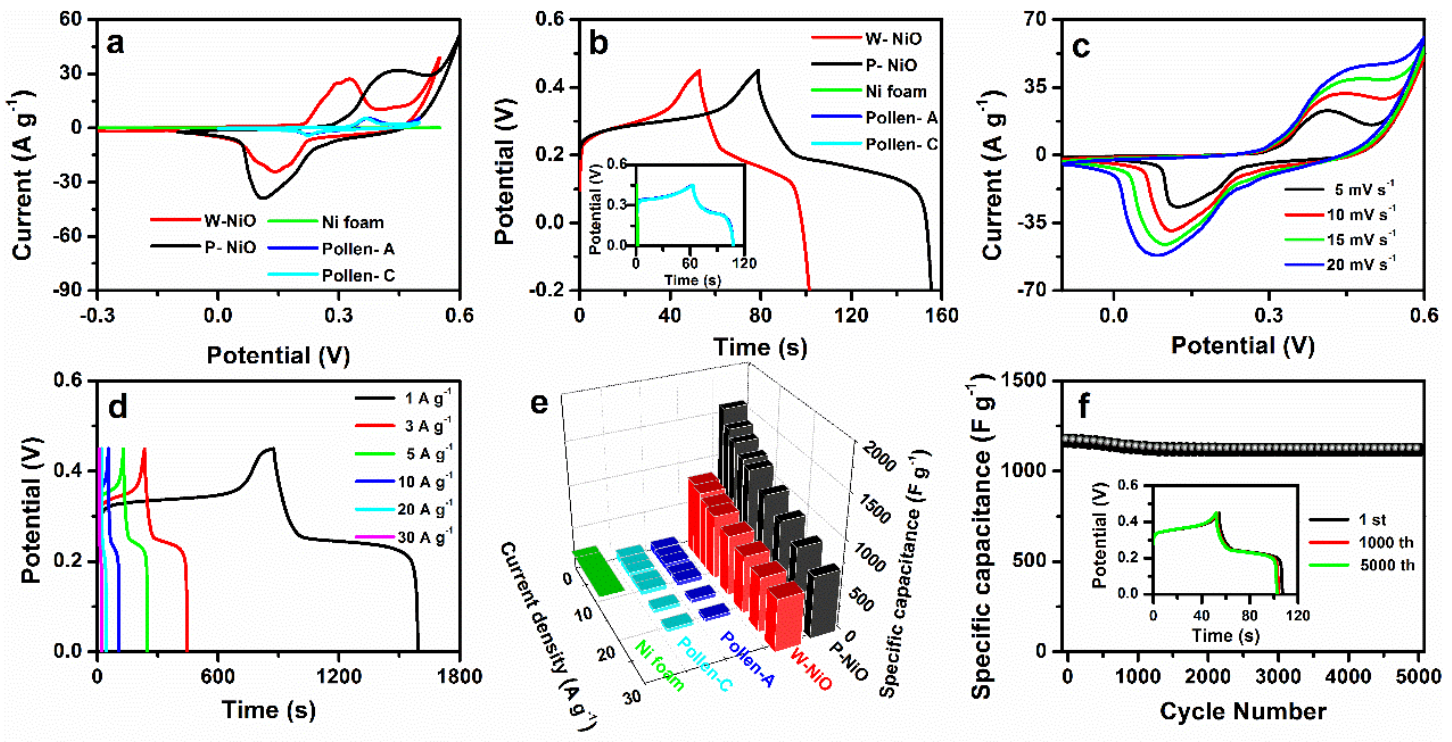

Figure 5 

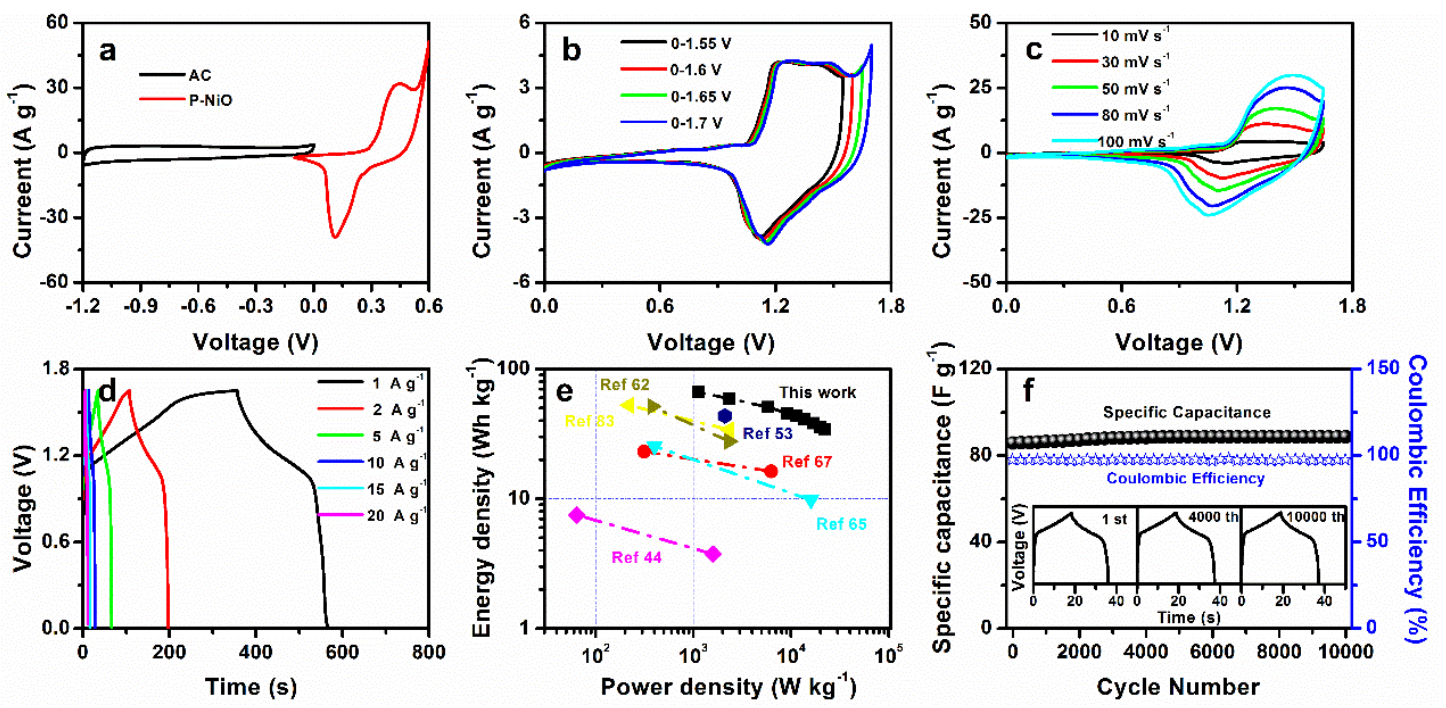

Figure 6 